\title{
Modeling of the Human Cerebral Collateral Circulation: Evaluation of the Impact on the Cerebral Perfusion in Case of Ischemic Stroke
}

https://doi.org/10.1515/cdbme-2019-0134

\begin{abstract}
Stroke is the third-most cause of death in developed countries. A new promising treatment method in case of an ischemic stroke is selective intracarotid blood cooling combined with mechanical artery recanalization. However, the control of the treatment requires invasive or MRI-assisted measurement of cerebral temperature. An auspicious alternative is the use of computational modeling. In this work, we extended an existing 1D hemodynamics model including the characteristics of the anterior, middle and posterior cerebral artery. Furthermore, seven ipsilateral anastomoses were additionally integrated for each hemisphere. A potential stenosis was placed into the M1 segment of the middle cerebral artery, due to the highest risk of occlusion there. The extended model was evaluated for various degrees of collateralization ("poor", "partial" and "good") and degrees of stenosis (0\%, 50\%, 75\% and $99.9 \%$ ). Moreover, cerebral autoregulation was considered in the model. The higher the degree of collateralization and the degree of stenosis, the higher was the blood flow through the collaterals. Hence, a patient with a good collateralization could compensate a higher degree of occlusion and potentially has a better outcome after an ischemic stroke. For a $99.9 \%$ stenosis, an increased summed mean blood flow through the collaterals of $+97.7 \%$ was predicted in case of good collateralization. Consequently, the blood supply via the terminal branches of the middle cerebral artery could be compensated up to $44.4 \%$ to the physiological blood flow. In combination with a temperature model, our model of the cerebral collateral circulation can be used for tailored temperature prediction for patients to be treated with selective therapeutic hypothermia.
\end{abstract}

Keywords: ischemic stroke, cerebral circulation, ipsilateral collaterals, M1 stenosis, hemodynamics model

\footnotetext{
*Corresponding author: Lorena Krames, Institute of Biomedical Engineering, Karlsruhe Institute of Technology (KIT), Karlsruhe, Germany, publications@ibt.kit.edu

Rosa Daschner, Yannick Lutz, Axel Loewe, Olaf Dössel, Institute of Biomedical Engineering, Karlsruhe Institute of Technology (KIT), Karlsruhe, Germany

Giorgio Cattaneo, Adceris GmbH \& Co KG, Pforzheim, Germany
}

\section{Introduction}

Therapeutic hypothermia (TH) can possibly lead to an improved outcome for ischemic stroke patients [1]. Currently, several techniques which induce $\mathrm{TH}$ of the whole body are used in case of ischemia. However, these can cause several side effects like pneumonia. In order to avoid these disadvantages, targeted temperature management (selective TH) can be used. This approach requires a detailed information of the cerebral circulation and a precise resolution of the brain temperature. In this work, we used a computational 1D hemodynamics model, which includes a detailed cerebral arterial and collateral structure to predict the cerebral blood flow distribution.

\section{Methods}

\subsection{Transmission Line Approach}

One main advantage of a 1D transmission-line approach is the real time capability for extensive fluid systems. For use in our case, the blood is regarded as an incompressible Newtonian fluid. Making use of a simplified form of the Navier-Stokes equation, two relations between pressure $p$ and flow $q$ can be established:

$$
\begin{gathered}
-\frac{\partial p}{\partial z}=\frac{\rho}{\pi r_{0}^{2}} \cdot \frac{\partial q}{\partial t}+\frac{8 \eta}{\pi r_{0}^{4}} \cdot q, \\
-\frac{\partial q}{\partial z}=\frac{3 \pi r_{0}^{3}}{2 E d} \cdot \frac{\partial p}{\partial t} .
\end{gathered}
$$

Due to analogies between the hydrodynamical and electrical systems, the coefficients of the differential equations per unit length $\Delta z$ can be defined as follows:

$$
R=\frac{8 \eta \Delta z}{\pi r_{0}^{4}}, L=\frac{\rho \Delta z}{\pi r_{0}^{2}}, C=\frac{3 \pi r_{0}^{3} \Delta z}{2 E d} .
$$

While the electric current corresponds to the flow, voltage represents the analog of the pressure. The coefficients can be derived from characteristics of the vessel (radius $r_{0}$, Young's modulus $E$ and wall thickness $d$ ) and from parameters of the fluid (dynamic blood viscosity $\eta=2.3 \cdot 10^{-3} \frac{\mathrm{kg}}{\mathrm{ms}}$ and density $\left.\rho=1020 \frac{\mathrm{kg}}{\mathrm{m}^{3}}\right)$. Moreover, the electrical resistance corresponds to the flow resistance, the inductance represents the inertia of the fluid and the capacity mirrors the compliance, a quantity for the elasticity of the vessel. For a discretization, the following differential relations for one short arterial segment can be 


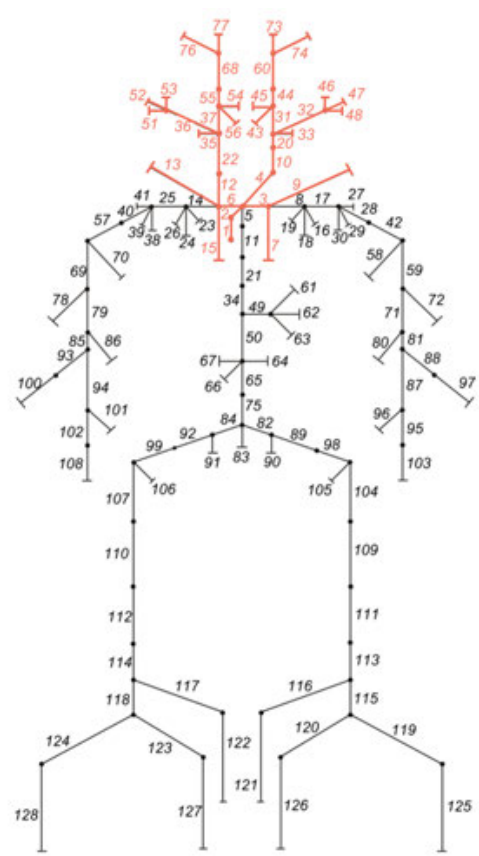

Fig. 1: Avolio's model with 128 segments [3]. Every segment is marked with a number. The highlighted segments are the part shown in Fig. 2.

used:

$$
\begin{gathered}
\frac{d p^{\text {out }}}{d t}=\frac{1}{C} \cdot\left(q^{\text {in }}-q^{\text {out }}\right), \\
\frac{d q^{i n}}{d t}=-\frac{R}{L}+\frac{1}{L} \cdot\left(p^{\text {in }}-p^{\text {out }}\right) .
\end{gathered}
$$

With series and parallel connections, in accordance to Kirchhoff's first law, it is possible to build a complete arterial network.

\subsection{Model of the Cerebral Arteries}

As a basic model, a multi-branched model by Avolio (see Fig. 1) was used [2]. In this model, the vessels of the human body were implemented in 128 segments. Later, Schwarz extended the model by taking an arterial ring structure into account, the Circle of Willis [3]. However, a more detailed model is required to investigate the impact of ipsilateral collaterals on the cerebral circulation. Therefore, we refined the strucutre of the three major cerebral arteries (anterior, middle and posterior cerebral artery (ACA, MCA, PCA)). Characteristics of the vessels were determined from literature. Since, the cerebral anatomy varies widely between individuals, we focused on the development of four standardized models depending on characteristics of the ACA and MCA. The most common structure for humans is a bifurcation of the MCA and an ACA with a typical callosomarginal artery [4]. The complete model is shown in Fig. 2. All simulations were performed in MATLAB/SIMULINK (2019a, The MathWorks, Natick, MA, USA).

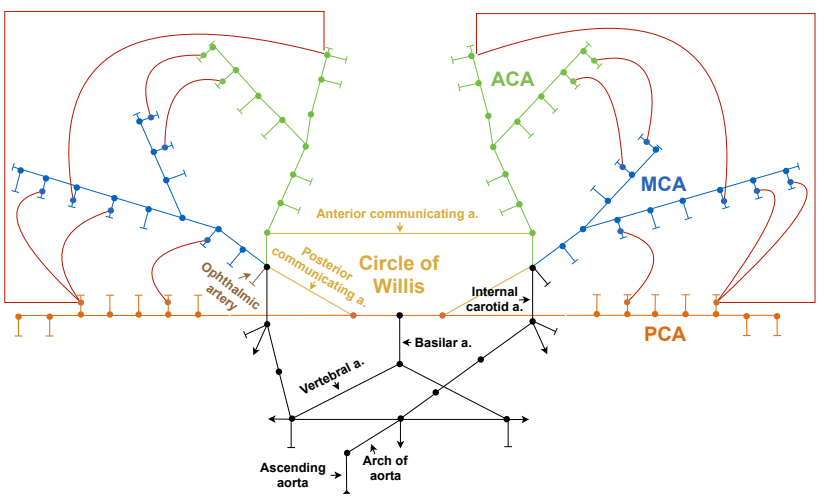

Fig. 2: Extended model of the cerebral circulation with ipsilateral collaterals, bifurcating MCA, and typical callosomarginal artery.

\subsection{Terminal Resistances}

The total periphery resistance is mainly governed by the resistance of the capillary network. The resistance of this fine branched network is defined by so-called terminal resistances. The values were calculated depending on the tissue volume and type of the supplied cerebral area of the respective artery using:

$$
q_{\text {term }}=v_{G M} \cdot V_{\text {term, } G M}+v_{W M} \cdot V_{\text {term }, W M}
$$

with $v_{G M}$ and $v_{W M}$ denoting the perfusion rates of grey (GM) and white matter (WM). $V_{\text {term }}=V_{\text {term, } G M}+V_{\text {term, } W M}$ represents the volume of the supply area of each terminal segment, which consists of an individual composition of WM and GM.

\subsection{Autoregulation}

Cerebral blood flow is regulated by the active change of the vessel lumen, caused by dilatation and constriction. Thus, a constant supply of the organs is ensured for changing blood flow conditions. In order to integrate this autoregulation, every terminal resistance has its own controller. The controller modifies the value of the terminal resistance by controlling the actual flow. In this process the flow averaged over time is compared to a reference flow $q_{\text {term }}$ (eq. 6). The mean value of the temporal flow is calculated by a third-order low-pass Butterworth filter $\left(f_{c}=0.1 \mathrm{~Hz}\right)$ and afterwards compared to a reference value. The block diagram of the autoregulation is shown in Fig. 3.

\subsection{Stenosis Model}

Considering the impact of ischemic stroke on the cerebral blood flow, we included an M1-stenosis. The stenosed artery is subdivided into three parts: the proximal part (in front of the stenosis), the stenosis part itself, and the distal part (behind the stenosis). The stenosis part is implemented as a single resistance. Moreover, the degree $S$ is variable and defined by the stenosis radius $r_{\text {sten }}=r_{0}\left(1-\frac{S}{100}\right)$, with $r_{0}$ equal to the initial M1-artery radius. In this work, four different degrees of 


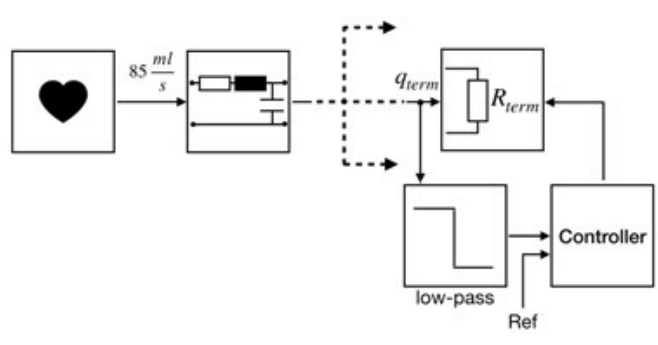

Fig. 3: Autoregulation of the terminal segments. The block on the left symbolizes the heart with a cardiac output of $85 \frac{\mathrm{ml}}{\mathrm{s}}$. The blood flow splits into lower flow rates depending on the arterial tree structure. Every terminal segment is concluded by a terminal resistance $R_{\text {term }}$ with a flow $q_{\text {term }}$. The autoregulation itself consists of a low-pass filter and a controller, which needs a reference value of the flow, and modifies the terminal resistance.

stenosis were considered ( $\mathrm{S}=0 \%$ (physiological case), $\mathrm{S}=50 \%$, $\mathrm{S}=75 \%$ and $\mathrm{S}=99.9 \%$ )

\subsection{Collateralization}

In case of an M1-stenosis, the blood supply through ipsilateral collaterals is particularly important. In a study by Vander Eecken and Adams, information about the occurrence and the size of the collaterals is given [5]. We included seven secondary collaterals between the main cerebral arteries in both hemispheres (3 ACA-MCA, 3 MCA-PCA, 1 ACA-PCA). To respect the high differences between individuals, the radii of the collaterals $r_{c o l}$ can be adapted by a factor $f$. In this work, the degree of collateralization is defined as $r_{c o l}=f \cdot r_{\text {initial }}$ with $f \in[1,1.5,2]$. The collaterals are also shown in Fig. 2 .

\section{Results}

\subsection{Physiological Case}

The physiological blood flow distribution of our model did not change markedly compared to the original model of Avolio and Schwarz. The deviations of the flow rate were $-4.3 \%$ (ACA), $-2.5 \%$ (MCA) and $-3.1 \%$ (PCA). Furthermore, in the healthy case, there was hardly any flow through the collaterals: $q_{\text {mean }}=0.03 \frac{\mathrm{ml}}{\mathrm{s}}$ (Fig. 5).

\subsection{Pathological Case}

The results can be divided into two different parts: First, the degree of collateralization was varied. In the second part, the degree of the M1-stenosis was varied. For the sake of clarity, only the flow rate in the right hemisphere will be presented throughout the following chapter. Moreover, only the MCA bifurcation and the ACA with typical callosomarginal artery is considered.

\section{Variation of Collateralization}

For this part, the degree of stenosis $S$ was constantly set to 99.9\%. In Fig. 4 the complete flow through the six collaterals of the MCA is shown. With an increasing degree of collateralization, the flow through the collaterals increased. The mean flow rates through the collaterals with the three different

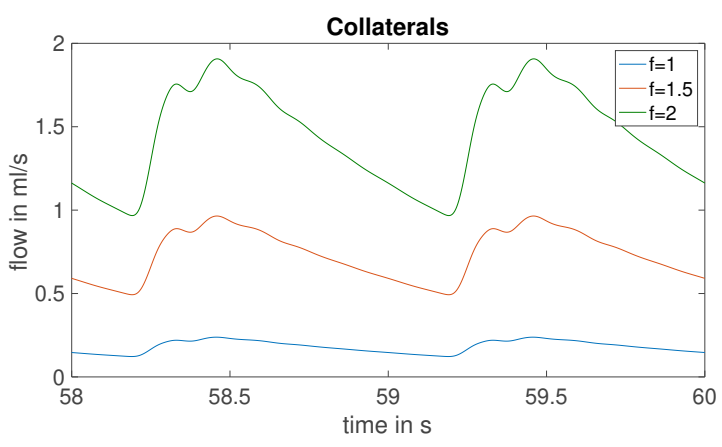

Fig. 4: Summed flow through the 3 ACA-MCA and 3 MCA-PCA collaterals in case of a $99.9 \%$ stenosis.

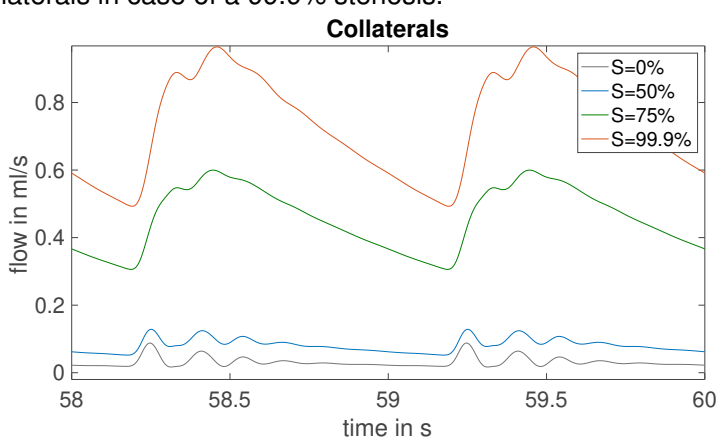

Fig. 5: Summed flow through the 3 ACA-MCA and 3 MCA-PCA collaterals with $r=1.5 \cdot r_{\text {initial }}$.

radii were: $q_{\text {mean.1r }}=0.18 \frac{\mathrm{ml}}{\mathrm{s}}, q_{\text {mean }} 1.5 \mathrm{r}=0.73 \frac{\mathrm{ml}}{\mathrm{s}}$ and $q_{\text {mean.2r }}=1.45 \frac{\mathrm{ml}}{\mathrm{s}}$. As there was no flow into the MCA from the carotid artery, the flow through the collaterals was equal to the flow into the terminal segments of the MCA branches. $6.9 \%$ (with $r_{1}$ ), $28.9 \%$ (with $r_{1.5}$ ) and $57.3 \%$ (with $r_{2}$ ) of the summed flow into the terminal segments of the MCA in the healthy case could be restored.

\section{Variation of Stenosis Degree}

For this investigation, the degree of collateralization was set to $f=1.5$. The stenosis degrees were $\mathrm{S}=50 \%, \mathrm{~S}=75 \%$ and $\mathrm{S}=99.9 \%$ and were compared to the physiological flow. Fig. 5 shows the total flow through the collaterals for the different degrees of stenosis. For increasing degree of stenosis, the flow through the collaterals increased. For a 50\% stenosis and a $75 \%$ stenosis, $+40.74 \%$ and $+72.1 \%$ of the mean flow rates through the collaterals in physiological case could be provided. In Fig. 6 the summed mean flow rates into the terminal segments of the MCA branches are depicted. The values for the flow into the terminal segments in case of a $50 \%$ and $75 \%$ stenosis were $2.46 \frac{\mathrm{ml}}{\mathrm{s}}$ and $1.69 \frac{\mathrm{ml}}{\mathrm{s}}$ respectively. Therefore, $97.2 \%$ (50\% stenosis) and $66.8 \%$ (75\% stenosis) of the physiological flow were preserved. For a $50 \%$ stenosis, the flow through the collaterals almost vanished and the summed flow into the MCA segments only slightly decreased (-2.8\%) compared to the reference flow. For a higher degree of stenosis $(75 \%)$, the flow into the terminal segments of the MCA 
branches decreased and the flow through the collaterals increased.

\section{Discussion}

We extended the 1D hemodynamics model by Avolio and Schwarz providing a higher level of detail of the cerebral arterial tree. Thanks to the extension, the blood flow distribution can be modeled more precisely. In the physiological case, the resulting flow rates were similar to the original model. Furthermore, the flow through the collaterals was almost zero, which is in accordance with literature [1].

In Fig. 6, all summed flows into the terminal segments of the MCA with different degrees of stenosis and collateralization are shown. For a $50 \%$ stenosis, the summed blood flow of the MCA did almost not change compared to the physiological case. Furthermore, different degrees of collateralization did not have a high impact on the blood supply. For higher degrees of stenosis (e.g 75\%), the blood supply of the MCA branches decreased more and more. In this case, the impact of the degree of collateralization was more significant. Higher degrees resulted in a higher perfusion through the ipsilateral collaterals. For a $99.9 \%$ stenosis, only the collaterals ensured a blood flow supply of the blocked MCA territory. Thus, different degrees of collateralization had significant impact on the blood flow. This especially shows the importance of the collateral circulation in a pathophysiological case. Summarizing, a higher degree of collateralization ensures a higher resulting blood supply in case of stenosis. Clinical data of the last few years consistently show a better outcome (reduced infarct size and faster recanalization) of patients with higher degree of collateralization [6]. Therefore, in accordance to these results, we categorize the degrees of collateralization as follows: $r=1 \cdot r_{\text {initial }}$ "poor" collateralization, $r=1.5 \cdot r_{\text {inital }}$ "partial" collateralization and $r=2 \cdot r_{\text {inital }}$ "good" collateralization. The collaterals allow an alternative route for the blood flow and can partly compensate the lack of supply. However, the efficiency of the collateral circulation also depends on age, duration of ischaemia and comorbidities.

\subsection{Limitations}

Literature data about the lengths of the cortical branches and the collaterals is limited. Therefore, we had to partly approximate these lengths based on the mean values of the other cerebral arteries. Moreover, the cerebral arterial tree varies largely between individuals. In this work, we focused on the model with the highest occurrence in humans [4]. The collateral circulation was divided into three different degrees to enable a more clear classification of the results.

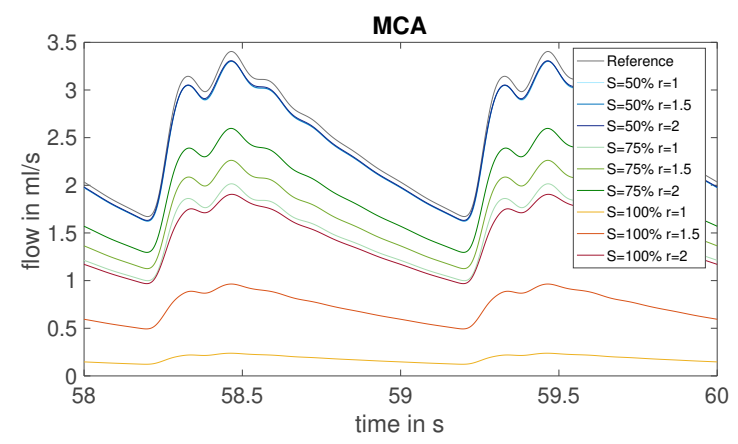

Fig. 6: Summed flow rates in the terminal MCA segments for three different degrees of stenosis and collateralization and for the physiological case (reference).

\section{Conclusion}

In this work, a 1D hemodynamics model was used to evaluate the cerebral collateral circulation in case of ischemic stroke. Due to regulation mechanims and secondary paths, humans are able to compensate ischemic strokes with low degree of stenosis. A "good" collateralization enables a significant compenstion of the blood supply. For a $99.9 \%$ stenosis the supply of MCA area is only ensured by ipsilateral collaterals. Therefore, collaterals have an important impact on the clinical outcome in case of a serious ischemic stroke. In literature, correlations between a well-marked collateral circulation and a better outcome after an ischemic stroke have been observed [7].

\section{Author Statement}

This study was supported by the German Federal Ministry of Economic Affairs and Energy (ZF436901AK6). Authors state no conflict of interest.

\section{References}

[1] N. Tariq and R. Khatri, "Leptomeningeal Collaterals in Acute Ischemic Stroke," J Vasc Interv Neurol, vol. 1, pp. 91-95, 2008

[2] A. P. Avolio, "Multi-branches model of the human arterial system," MBEC, vol. 18, pp. 709-718, 11980.

[3] M.Schwarz, "Modellbasierte Operationsplanung und Ueberwachung hypothermer Patienten," KIT Scientific Publishing, 2009.

[4] F.Umansky, S.M.Juarez, M.Dujovny, et al., "Microsurgical anatomy oft he proximal segments of the middle cerebral artery," Journal of Neurosurgery, vol. 61, pp. 458-467, 1984.

[5] H. M. Vander Eecken and R. D. Adams, "The anatomy and functional significance of the meningeal arterial anastomoses of the human brain," Journal of neuropathology and experimen- tal neurology, vol. 12, pp. 132-157, 1953.

[6] F. McVerry, D. Liebeskind, and K. Muir, "Systematic review of methods for assessing leptomeningeal collateral flow," American Journal of Neuroradiology, vol. 33, pp. 576-582, 2012.

[7] D. S. Liebeskind, "Collateral circulation," Stroke, vol. 34, pp. 2279-2284, 2003. 